\title{
Energy and mode filtering in a graphene channel with unevenly spaced barriers with a smooth profile
}

\author{
Paolo Marconcini \\ Dipartimento di Ingegneria dell'Informazione, Università di Pisa, \\ Via Girolamo Caruso 16, 56122 Pisa, Italy
}

Received: May 9, 2020. Revised: June 7. Accepted: June 8, 2020. Published: June 9, 2020

\begin{abstract}
We simulate the transport and shot noise behavior of graphene armchair ribbons with a series of parallel, unevenly spaced potential barriers with a smooth profile (which could result from the electrostatic effect of negatively biased gates). We analyze the effect of Klein tunneling and resonant tunneling on the individual modes propagating through the graphene channel, showing that this structure can behave as a mode and an energy filter for the charges injected from the contacts. Moreover, we study the different transport regimes (ballistic, strong localized, and diffusive) that can take place inside the graphene ribbon and the effect on the shot noise behavior of the device.
\end{abstract}

Keywords-Graphene, ribbon, barriers, conductance, shot noise, Klein tunneling, resonant tunneling.

\section{INTRODUCTION}

$\mathbf{I}$ $\mathrm{N}$ the last few years, a large interest has developed on graphene and related two-dimensional materials [1]-[20]. Graphene is a recently isolated and characterized material made up by an planar hexagonal lattice of carbon atoms with $s p^{2}$ hybridization. It is transparent and flexible and presents very interesting properties, among which high mechanical strength and electrical and thermal conductivity [21]-[23]. Its high mobility, planarity and one-atom thickness have suggested a possible application in electronic devices [24]-[30], especially for high-speed analog electronics and for sensors [31]-[33]. At low energies, the transport behavior of graphene can be described in terms of four envelope functions $F_{\beta}^{\vec{\alpha}}(y)$ (with $\vec{\alpha}=\vec{K}, \vec{K}^{\prime}, \beta=A, B$ ), corresponding to the two inequivalent atoms $A$ and $B$ of the lattice unit cell and to the two inequivalent degeneration points (Dirac points) $\vec{K}$ and $\vec{K}^{\prime}$ of the graphene dispersion relations. The envelope functions have to satisfy the Dirac-Weyl equation [34], i.e., the same relation which governs the relativistic behavior of massless $1 / 2$-spin particles. This has made it possible to observe in graphene, at non-relativistic speeds, relativistic phenomena such as Klein tunneling (a physical phenomenon according to which a relativistic particle orthogonally impinging against a barrier is able to transmit across it with unit probability) [35][37].

Several research groups have explored the transport behavior of graphene in the presence of potential barriers. However, these studies have mainly focused on unconfined graphene with one or a series of periodically placed barriers (i.e.,

Invited Paper.

This work was partially supported by the Italian Ministry of Education and Research (MIUR) in the framework of the CrossLab project (Departments of Excellence).

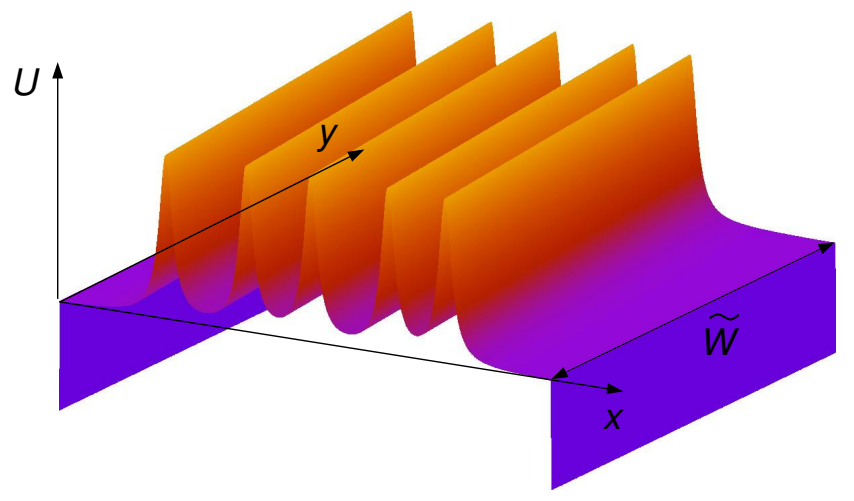

Fig. 1. Sketch of the potential energy considered in our analysis, made up by a series of parallel tunnel barriers with a Lorentzian profile, with different interbarrier distances.

with a superlattice) [38]-[54] or, in a few cases, on confined graphene with a single tunnel barrier [55]-[59]. Instead, here, starting from Ref. [12] but considering barriers with a more realistic, smooth (in particular Lorentzian) profile, we numerically analyze the transport and shot noise behavior of a graphene armchair ribbon with a series of parallel potential barriers located at different reciprocal distances (a sketch of the considered potential profile is shown in Fig. 1). These smooth tunnel barriers can derive for example from the electrostatic action of parallel gates, negatively biased and separated from the graphene transmission channel by a dielectric layer [60][62]. We will show that transport is mainly dominated by Klein tunneling and by resonant tunneling, which give rise to mode filtering and to energy filtering, respectively. In our study, we will also analyze how the geometry of the structure can affect the observed behavior, and in particular the transport regime inside the device.

\section{NUMERICAL METHOD}

For our analysis, we have used an envelope-function based code that we have developed to study transport in graphene armchair structures with a generic potential [63]. This numerical approach is valid in the low energy range, where the graphene dispersion relations are approximately linear, and if the wave function and the potential are slow-varying on the scale of the lattice spacing [63]. Although this continuum description is not as detailed as an atomistic model, it is preferable when a structure with a large number of atoms has to be simulated, due to its higher numerical efficiency. The simulator 
is based on the numerical solution of the Dirac-Weyl equation (the equation that the four envelope functions of graphene have to satisfy [34]), through a scattering matrix iterative procedure. Let's call $x$ and $y$ the longitudinal (transport) direction and the transverse direction, respectively, and $\tilde{W}$ the effective width of the ribbon (Fig. 1).

In our numerical procedure, we divide the device into a series of sections inside each of which the potential is approximately independent of the longitudinal coordinate $x$. The first step is the solution of the Dirac-Weyl equation inside each section. In such a domain, due to translational invariance, each of the four envelope functions can be written as the product of a transverse component depending only on $y$ and a plane wave propagating along $x: F_{\beta}^{\vec{\alpha}}(x, y)=\Phi_{\beta}^{\vec{\alpha}}(y) \exp \left(i \kappa_{x} x\right)$ (notice that the envelope functions depend on the wave vector $\vec{\kappa}$ measured with respect to the Dirac points). With this position, the Dirac-Weyl equation becomes a differential eigenproblem (in the transverse components $\Phi_{\beta}^{\vec{\alpha}}(y)$ of the envelope functions and in the longitudinal wave vector $\kappa_{x}$ ) on a one-dimensional domain, corresponding to $y \in[0, \tilde{W}]$. The boundary conditions are enforced by the vanishing, at the edges of the ribbon ( $y=0$ and $y=\tilde{W})$, of the total electron wave function, expressed in terms of the four envelope functions. More in detail, the problem has the form:

$$
\left\{\begin{array}{l}
\left(\sigma_{z} \partial_{y}+\sigma_{x} f(y)\right) \vec{\varphi}^{\vec{K}}(y)=-\kappa_{x} \vec{\varphi}^{\vec{K}}(y) \\
\left(-\sigma_{z} \partial_{y}+\sigma_{x} f(y)\right) \vec{\varphi}^{\vec{K}^{\prime}}(y)=-\kappa_{x} \vec{\varphi}^{\vec{K}^{\prime}}(y) \\
\vec{\varphi}^{\vec{K}}(0)=\vec{\varphi}^{\prime}(0) \\
\vec{\varphi}^{\vec{K}}(\tilde{W})=e^{2 i K \tilde{W}} \vec{\varphi}^{\vec{K}^{\prime}}(\tilde{W}),
\end{array}\right.
$$

where: $\sigma_{x}, \sigma_{y}, \sigma_{z}$ are the Pauli matrices, $\partial_{y}=d / d y$,

$$
\vec{\varphi}^{\vec{K}}(y)=\left[\begin{array}{c}
\Phi_{A}^{\vec{K}}(y) \\
\Phi_{B}^{\vec{K}}(y)
\end{array}\right], \vec{\varphi}^{\vec{K}^{\prime}}(y)=i\left[\begin{array}{c}
\Phi_{A}^{\vec{K}^{\prime}}(y) \\
\Phi_{B}^{\vec{K}^{\prime}}(y)
\end{array}\right],
$$

and $f(y)=(U(y)-E) /\left(v_{F} \hbar\right)$. $U$ is the potential energy, $E$ is the injection energy, $v_{F}$ is the graphene Fermi velocity, $\hbar$ is the reduced Planck constant, $K=4 \pi /(3 a)$ if the ordinate of a Dirac point, and $a$ is the graphene lattice constant. The solution through standard techniques of this problem in the direct domain is known to suffer numerical problems (in particular, fermion doubling [63]-[65]). However, with these definitions [63], [66], [67]:

$$
\vec{\varphi}(y)= \begin{cases}\vec{\varphi}^{\vec{K}}(y) & y \in[0, \tilde{W}] \\ e^{i 2 K \tilde{W}} \vec{\varphi}^{\vec{K}^{\prime}}(2 \tilde{W}-y) & y \in[\tilde{W}, 2 \tilde{W}],\end{cases}
$$

the system can be reformulated as the following equivalent eigenproblem on the domain $[0,2 \tilde{W}]$, with periodic boundary conditions at the domain edges for the function $e^{-i K y} \vec{\varphi}(y)$ :

$$
\left\{\begin{array}{l}
\left(\partial_{y} \sigma_{z}+\tilde{f}(y) \sigma_{x}\right) \vec{\varphi}(y)=-\kappa_{x} \vec{\varphi}(y) \\
e^{-i 2 K \tilde{W}} \vec{\varphi}(2 \tilde{W})=\vec{\varphi}(0)
\end{array}\right.
$$

where $\tilde{f}(y)=f(\tilde{W}-|\tilde{W}-y|)$. This problem can be very efficiently solved in the reciprocal domain, avoiding the fermion doubling problem and obtaining a basis of transport modes in each of the sections in which we have divided the device. The next step is the calculation of the scattering matrix which relates adjacent sections, i.e., the set of transmission and reflection coefficients that describes the transport across the interface between the two sections. In order to compute such a quantity, we enforce the continuity, across the interface, of the wave fuction (and thus of its components on the two graphene sublattices made up by all the $A$-type atoms and all the $B$-type atoms). Imagining to inject each mode at the time across the interface and projecting the resulting equations onto a basis of sine functions, we obtain a linear system in the transmission and reflection coefficients, that can be easily solved using linear algebra routines. The successive step is the composition (through standard procedures [68]) of the scattering matrices of the different parts of the device, in such a way as to obtain the scattering matrix of the overall device, and thus its transmission matrix $t$. Finally, from the transmission matrix $t$ it is possible to compute the conductance $G$, the shot noise power spectral density $S_{I}$, and the Fano factor $F$ (i.e., the ratio between the actual shot noise power spectral density $S_{I}$ and the value $2 e I$ that would be present if the charge carriers moved independently one from the others, with $e$ is the elementary charge and $I$ the current flowing through the device). Indeed, according to the Landauer-Büttiker formulas [69]-[71]:

$$
\begin{gathered}
G=\frac{2 e^{2}}{h} \sum_{i} w_{i}, \quad S_{I}=\frac{4 e^{3}}{h} V \sum_{i} w_{i}\left(1-w_{i}\right), \\
F=\frac{S_{I}}{2 e I}=\frac{\sum_{i} w_{i}\left(1-w_{i}\right)}{\sum_{i} w_{i}},
\end{gathered}
$$

where $h=2 \pi \hbar$ is Planck's constant, the $w_{i}$ 's are the eigenvalues of the matrix $t^{\dagger} t, I=G V$ (with $V$ the externally applied voltage), and the sums are done over all the modes propagating in the input lead.

The values of $G$ and $S_{I}$ obtained from Eq. (5) should be averaged over all the energy domain, weighting the result at each energy with the difference between the Fermi-Dirac occupation functions at the input and at the output leads. However, in our simulations we assume to operate at a temperature $T$ such that $K_{B} T<<e V$ (with $K_{B}$ the Boltzmann constant), in such a way as to neglect the contribution of thermal noise. Therefore, the conductance and shot noise power spectral density will be uniformly averaged between the electrochemical potentials of the two leads (which differ by $e V$ ). In the case of the Fano factor $F$ (Eq. (6)), the average has to be performed separately on the numerator and on the denominator, which are the directly measurable quantities.

The presence of the contacts is modeled in the simulations including two sections with negative potential energy $(-0.2 \mathrm{eV}$ in our simulations) at the input and output leads (Fig. 1), which allow the injection of a sufficiently high number of propagating modes in the structure, for all the considered values of injection energy.

We have developed the code in Fortran, exploiting Lapack linear algebra routines [72]. The simulator has already been successfully used to simulate the behavior of several graphenebased devices and structures and to reproduce experimental result, helping to explain the observed results [12]-[14], [60], 
[61], [63], [66]. The validity of our code has been previously tested comparing its results with those obtained with a tightbinding based code [63]. Moreover, its results for the case of a single rectangular barrier agree with those reported in Refs. [38], [73].

\section{NUMERICAL RESULTS}

Here we simulate the transport behavior of armchair graphene ribbons with parallel potential barriers (in such a way as to preserve Klein tunneling).

We consider potential barriers with a smooth, Lorentzian profile, of the kind $U(x)=U_{M} /\left\{1+\left[\left(x-x_{0}\right) / H\right]^{2}\right\}$, where $U_{M}$ is the maximum value, $x_{0}$ is the central position, and $H$ is the half-width at half-maximum. This represents an approximation of the actual potential profile electrostatically generated at the graphene level by negatively biased gates (Fig. 1). Smooth barriers like the considered ones are known to present a quite selective Klein tunneling behavior [40], [48].

In the case of parallel and equally spaced barriers, when the structure becomes periodic, the transport behavior is dominated by resonant tunneling through the states quasi-localized in the regions between adjacent barriers, and it has been found that extra Dirac points appear in the graphene spectrum [49][54].

Here, instead, the barriers are assumed to be parallel, but unevenly spaced: in particular, in our simulations the interbarrier distances are randomly extracted, with uniform probability distribution, inside a range of possible values (which we will specify in the following).

Armchair graphene ribbons can present either a semiconducting or a metallic behavior, depending on the exact number $N$ of dimer lines across their width: if $N$ can be written as $3 M-1$, with $M$ an integer number, the ribbon is metallic, otherwise it is semiconducting. Indeed, the wave function in the graphene ribbon has to satisfy Dirichlet boundary conditions at the edges; this enforces a discretization on the transverse component of the wave vector, which can only assume values multiple of $\pi / \tilde{W}$. If $N=3 M-1$ one of these values coincides with the ordinate in the reciprocal space of the Dirac points (the degeneration points of the graphene dispersion relations) and thus the ribbon presents a zero energy gap. Instead, if $N \neq 3 M-1$ this does not happen and the energy gap is different from zero.

In our analysis, we will consider both the case of a semiconducting ribbon, and that of a metallic ribbon, in the presence of unevenly-spaced parallel Lorentzian barriers with identical or different shape, orthogonal or tilted with respect to the ribbon edges.

We assume to apply a potential $V=0.4 \mathrm{mV}$ between the output and input contacts and, therefore, we average our results over a number of energies (5 in our simulations, which are sufficient, due to the very small variation of the results on the considered energy range) uniformly distributed over a $0.4 \mathrm{meV}$ interval.

We have first analyzed the case of a semiconducting ribbon with unevenly spaced identical Lorentzian barriers, orthogonal to the ribbon edges. In particular, we have considered a ribbon
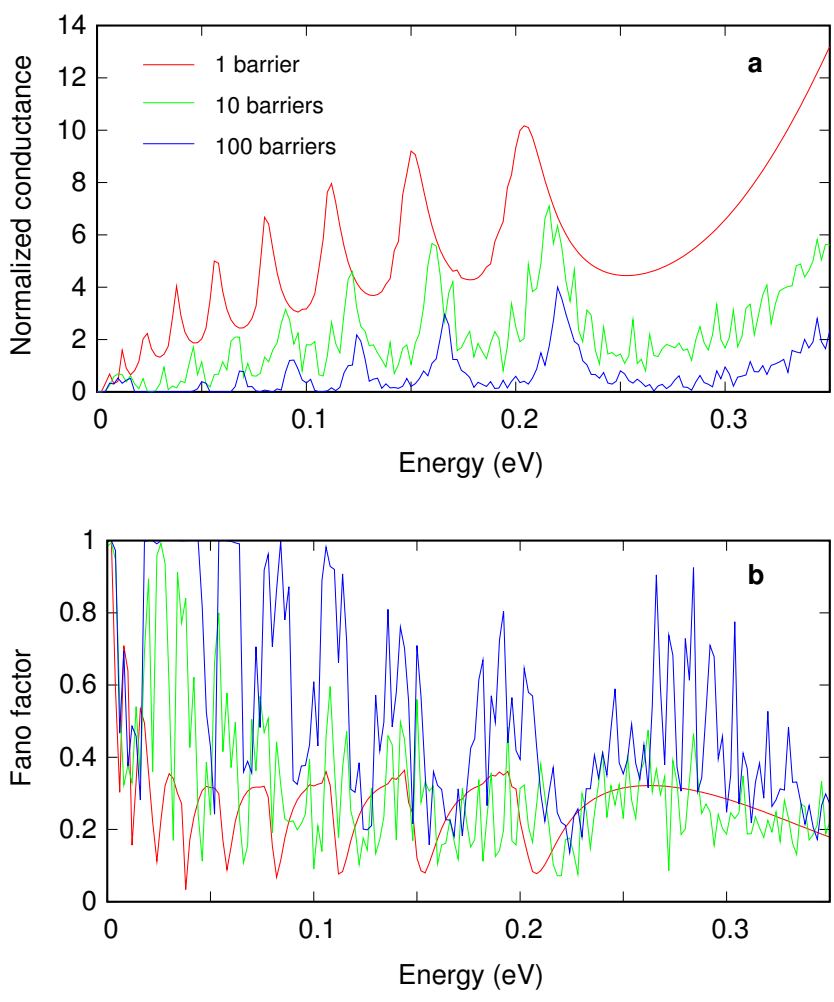

Fig. 2. (a) Normalized conductance $G / G_{0}$ (where $G_{0}$ is the conductance quantum) as a function of the injection energy for a semiconducting graphene ribbon with 1626 dimer lines, with a series of 1,10 , and 100 tunnel barriers (orthogonal to the ribbon edges) with identical Lorentzian profile and with different interbarrier distances. (b) Corresponding behavior of the Fano factor as a function of the injection energy.

with 1626 dimer lines (and thus approximately $200 \mathrm{~nm}$ wide), barriers with $U_{M}=0.3 \mathrm{eV}$ and $H=20 \mathrm{~nm}$, and values of the interbarrier distances between $150 \mathrm{~nm}$ and $250 \mathrm{~nm}$.

In Fig. 2(a) we show the results for the normalized conductance $G / G_{0}$ (where $G_{0}=2 e^{2} / h$ is the conductance quantum) as a function of the injection energy $E$ that we have obtained for 1, 10, and 100 barriers. In Fig. 2(b) we report the corresponding values of the Fano factor $F$.

In the case of a single barrier, we notice that the conductance is zero for $E=0$, due to the absence of any propagating mode at zero energy in a semiconducting ribbon. Increasing $E$, the conductance presents several maxima. For the energy corresponding to the barrier peak, the conductance has a nonzero value, because transmission takes place through the narrow barrier through evanescent modes. Regarding the Fano factor, we observe that it has minima for the energies for which the conductance presents peaks, as a consequence of the relation between the two quantities, given by Eq. (6) (with $I=G V)$. Moreover, for $E=0$ the value of the Fano factor is 1 (when transmission is low, carriers move about independently, and thus there is no shot noise suppression), while near the top of the barrier the Fano factor is $1 / 3$ (as predicted by Tworzydło et al. for the transmission by evanescent modes through a narrow graphene sample [15]).

Increasing the number of barriers, for every energy the conductance progressively decreases; at the same time, we observe an increase of the relative variation between the local 
minima and the local maxima. Looking at the Fano factor behavior, we notice that the fluctuations as a function of energy increase, as a consequence of the interference between the complex quantum paths in the device. Finally, for a sufficiently high number of barriers, we notice that the conductance goes to zero and the Fano factor approaches 1 (absence of shot noise suppression), as a consequence of the vanishing of the transmission through the structure.

Since the barriers are orthogonal to the ribbon edges and thus to the transmission direction $x$, they do not introduce mixing among the modes flowing through the device. Therefore, the transmission matrix of the structure is diagonal, with each diagonal element corresponding to a single mode. In order to gain a better insight into the transport phenomena, it is useful to separately examine the contribution of each mode.

Each mode $i$ is identified by one of the values allowed (i.e., which satisfy the Dirichlet boundary conditions at the ribbon edges) of the transverse component of the wave vector, and thus by the difference $\kappa_{y i}$ between this component and the ordinate in the reciprocal space of the Dirac point. We order the modes for increasing $\left|\kappa_{y i}\right|$. Outside the barriers, where the potential energy is nearly zero, the relationship between the energy value $E$ and the components $\kappa_{x i}$ and $\kappa_{y i}$ of the wave vector (measured from the Dirac point) of the $i$-th mode is $E= \pm \hbar v_{F} \sqrt{\kappa_{x i}^{2}+\kappa_{y i}^{2}}$. The angle of incidence of the mode against the barrier is given by the angle of $\vec{\kappa}$ with respect to the $x$ direction, i.e., $\theta_{i}=\arctan \left(\kappa_{y i} / \kappa_{x i}\right)$. Each barrier represents a finite potential well for the holes. Therefore, for each value of the transverse wave vector (and thus for each mode $i$ ) a discrete number of quasi-bound states $\mathcal{E}_{i, m}$ exists inside each barrier, due to the longitudinal confinement produced by the barrier.

In Fig. 3 we report (with a color map) the value of the transmission $w_{i}$ that we have numerically evaluated for each single mode as a function of the injection energy $E$ and of the mode index $i$. In the panel (a) we show the results for a single barrier, while in the panel (b) we plot those for 10 identical cascaded barriers.

We notice that increasing $i$ the average value of the transmission value decreases. This is due to Klein tunneling: modes with a lower order $i$ have a lower $\left|\kappa_{y i}\right|$ (and, for a fixed $E$, a higher $\left.\left|\kappa_{x i}\right|\right)$. Therefore, these modes have a smaller $\left|\theta_{i}\right|$, i.e. they impinge more orthogonally against the barrier. Due to Klein tunneling, these modes have a larger probability to pass through the barrier. Therefore, a series of barriers could serve as a mode filter, since only the lowest-order modes would be able to pass through a finite number of barriers. Let us notice that for a mode with $\theta_{i}=0$ the transmission probability would be 1; however, in a semiconducting ribbon no mode has $\kappa_{y i}=0$ (and thus has $\theta_{i}=0$ and unit transmission) because none of the allowed transverse wave vectors is equal to the ordinate (in the reciprocal space) of the Dirac points. Therefore, sufficiently increasing the number of barriers, all the modes, including the lowest ones, will be suppressed.

The other main effect that appears from Fig. 3 is the presence of energy ranges (which depend also on the mode) for which the transmission is higher. This effect can be attributed
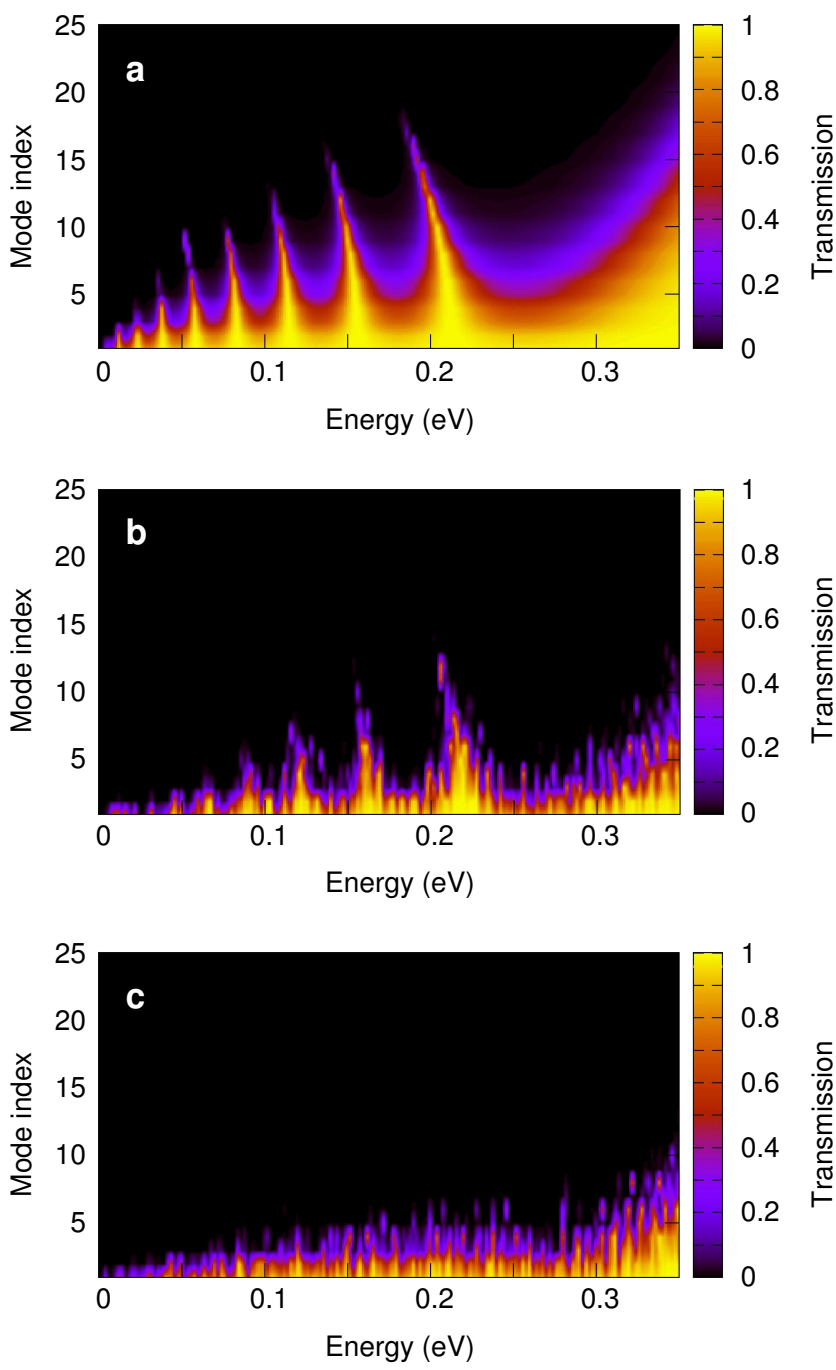

Fig. 3. (a) Mode transmission as a function of the injection energy and of the mode index, for a semiconducting graphene ribbon (with 1626 dimer lines) with a single tunnel barrier (a), 10 identical barriers (b), and 10 different barriers (c). The barriers are orthogonal to the ribbon edges, have a Lorentzian profile, and are unevenly spaced.

to resonant tunneling: the generic mode $i$ has a larger probability to transmit through the barriers for the energies $\mathcal{E}_{i, m}$ of the states quasi-localized inside the barriers corresponding to that mode. Since in this case we are considering identical barriers, the values of these energies $\mathcal{E}_{i, m}$ are the same for all the barriers. Therefore, increasing the number of barriers, while the average conductance decreases, this energy selection strengthens, since each barrier performs a further selection on the output of the previous ones. Therefore, a series of several identical barriers acts as an energy filter, which allows tunneling only for specific intervals of energies.

The total conductance shown in Fig. 2(a) is given by the sum of the contributions of all the propagating modes and thus is affected by the described tunneling phenomena. Since we are considering a semiconducting ribbon, with a nonzero energy gap, for $E=0$ no mode is able to transmit. Moreover, since no mode has $\kappa_{y i}=0$ and thus impinges orthogonally against the barriers, all the modes are affected by the barriers and are progressively suppressed: therefore, sufficiently increasing 

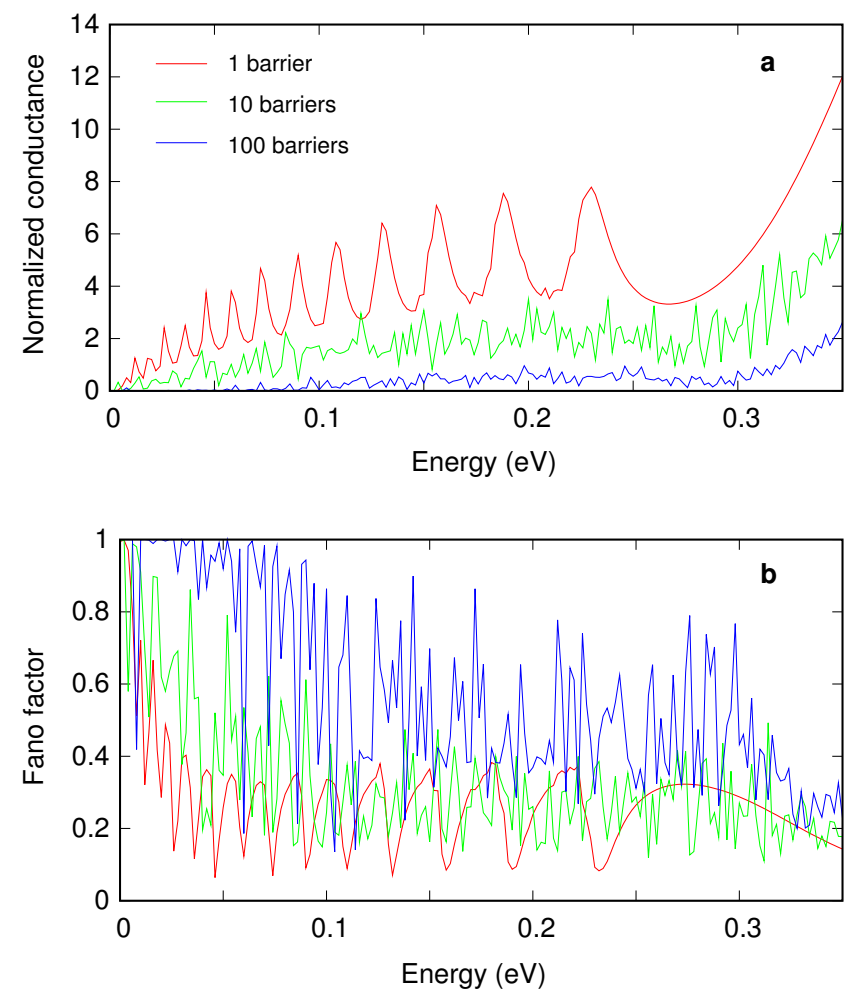

Fig. 4. (a) Normalized conductance $G / G_{0}$ as a function of the injection energy for a semiconducting graphene ribbon with 1626 dimer lines, with a series of 1,10 , and 100 tunnel barriers (orthogonal to the ribbon edges) with different Lorentzian profile and with different interbarrier distances. (b) Corresponding behavior of the Fano factor as a function of the injection energy.

the number of barriers, the conductance vanishes (and thus Fano factor approaches 1). As a consequence of resonant tunneling, the conductance is larger for certain intervals of energies (as we have noticed); the position of the maxima can be approximately estimated evaluating the energies of the states quasi-bound inside the barriers for the lowest-order mode, which is the mode for which transmission is maximum and thus which more contributes to the overall conductance.

Then, we have simulated the transport and shot noise behavior of the same armchair semiconducting ribbon with 1626 dimer lines, in which however the unevenly spaced barriers (with interbarrier distances randomly distributed between $150 \mathrm{~nm}$ and $250 \mathrm{~nm}$, as in the previous case) are not identical. In detail, all the barriers have a Lorentzian profile with a $0.3 \mathrm{eV}$ peak, but their half-width at half-maximum is randomly extracted between $2.5 \mathrm{~nm}$ and $37.5 \mathrm{~nm}$. The behavior of the normalized conductance as a function of the injection energy is shown in Fig. 4(a) for 1, 10, and 100 barriers, while in Fig. 4(b) we report the corresponding results for the Fano factor. Since the ribbon is still semiconducting, for $E=0$ no mode propagates through the device, the conductance is zero and the Fano factor is 1 (as in the previous case). However, examining the behavior as a function of energy we notice that increasing the number of barriers the conductance peaks (and the corresponding oscillatory behavior for the Fano factor), observed for a single barrier, disappear. The situation is better understood analyzing the transmission as a function of the
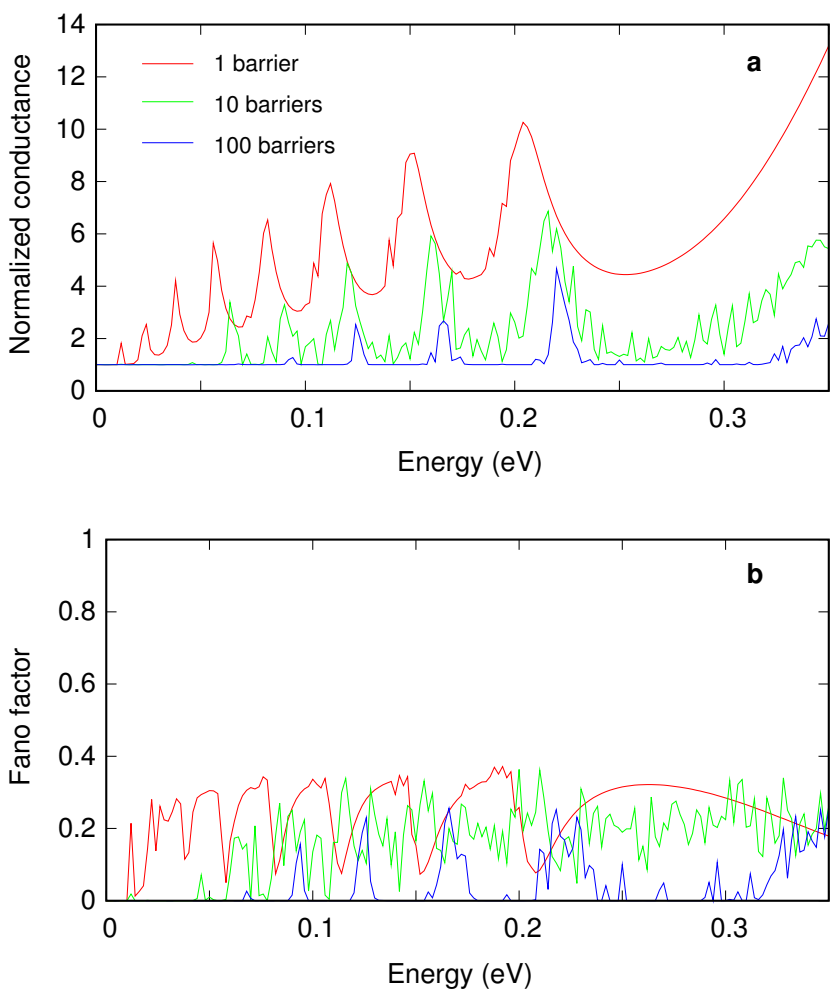

Fig. 5. (a) Normalized conductance $G / G_{0}$ as a function of the injection energy for a metallic graphene ribbon with 1625 dimer lines, with a series of 1,10 , and 100 tunnel barriers (orthogonal to the ribbon edges) with identical Lorentzian profile and with different interbarrier distances. (b) Corresponding behavior of the Fano factor as a function of the injection energy.

injection energy for each separate mode flowing through the structure: in the panel (c) of Fig. 3 we report a color plot for the case of 10 different cascaded barriers. We notice that Klein tunneling remains: the lowest-order modes are those experiencing the largest transmission, while modes with higher order are more suppressed by the barriers. Since the ribbon is still semiconducting, no mode is exactly orthogonal to the barriers and thus passes unaltered through the structure. Therefore, for a sufficiently high number of barriers, no mode is able to transmit through the structure, the conductance vanishes and the value of the Fano factor approaches 1 . However, in the color map of Fig. 3(c) we do not notice any more favored energies for which transmission is higher. Indeed, since the barriers are different, also the energies $\mathcal{E}_{i, m}$ of their quasi-localized states are not the same. Therefore, no energy exists for which resonant tunneling is possible through all the barriers. Due to the absence of resonant tunneling, the peaks in the conductance as a function of energy disappear.

We have then considered the case of a metallic armchair ribbon, in particular with 1625 dimer lines. First, we have simulated the behavior in the presence of a series of identical Lorentzian barriers (with height $U_{M}=0.3 \mathrm{eV}$ and half-width at half-maximum $H=20 \mathrm{~nm}$ ), with interbarrier distances randomly distributed between $150 \mathrm{~nm}$ and $250 \mathrm{~nm}$ (the same case already considered in the semiconductor ribbon). In Fig. 5(a) and Fig. 5(b) we show the behavior of the conductance and of the Fano factor as a function of energy, respectively. Instead, in Fig. 6(a) and Fig. 6(b) we plot the value of the transmission 

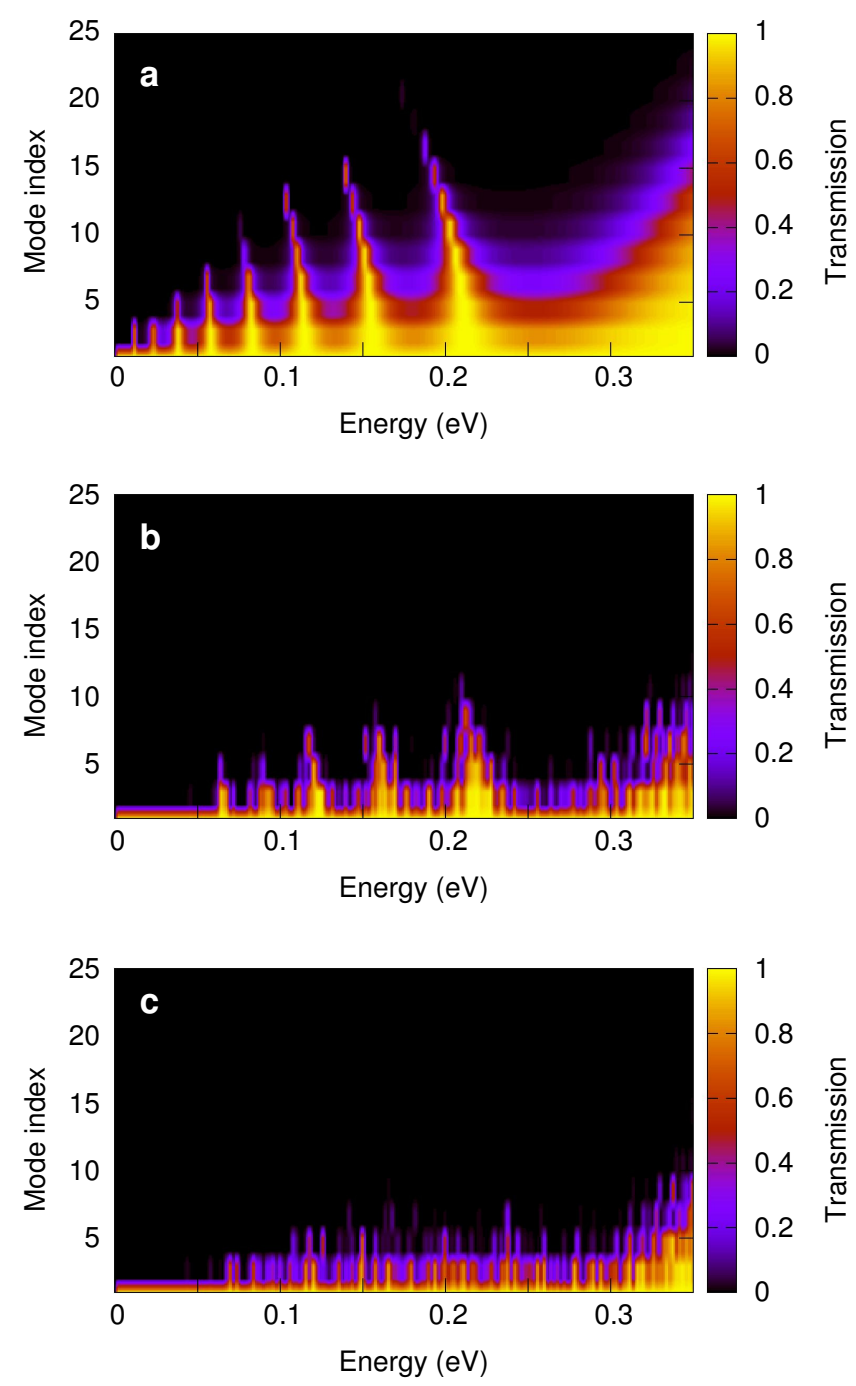

Fig. 6. (a) Mode transmission as a function of the injection energy and of the mode index, for a metallic graphene ribbon (with 1625 dimer lines) with a single tunnel barrier (a), 10 identical barriers (b), and 10 different barriers (c). The barriers are orthogonal to the ribbon edges, have a Lorentzian profile, and are unevenly spaced.

for the individual modes as a function of the energy and of the mode index, for a single barrier and for a series of 10 identical barriers, respectively. Also in this case, we notice the effect of Klein tunneling, with the lowest-order modes being transmitted by the device with higher transmission probability, and of resonant tunneling, with the transmission probability much higher at the energies $\mathcal{E}_{i, m}$ corresponding to the states quasi-localized inside the barriers. However, contrary to semiconducting ribbons, in a metallic ribbon one of the transverse wave vectors which satisfy the Dirichlet boundary conditions at the ribbon edges coincides with the ordinate (in the reciprocal space) of the Dirac point. Therefore, the lowestorder mode has $\kappa_{y i}=0$. This mode is able to propagate in a metallic ribbon already from $E=0$ and, having an angle of incidence $\theta_{i}=0$, impinges orthogonally against the barriers and thus transmits perfectly through the device at every energy. The consequences can be clearly noticed in the behaviors of the conductance and of the Fano factor. Contrary to the case of
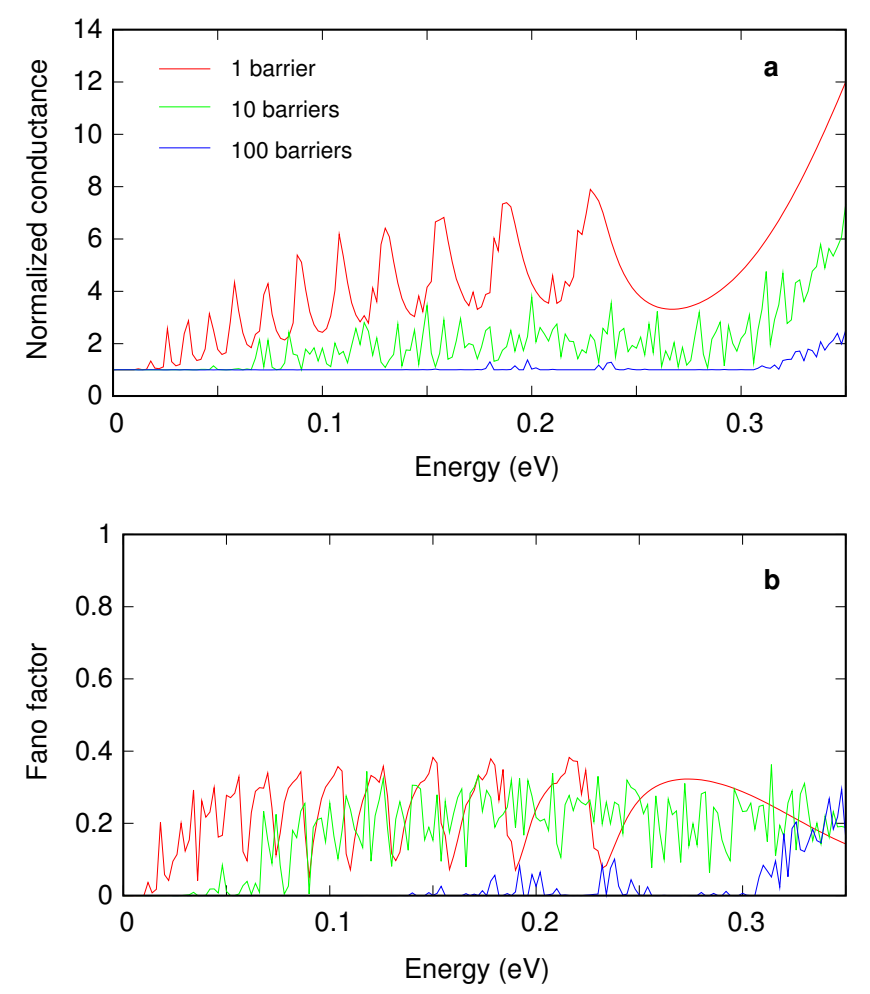

Fig. 7. (a) Normalized conductance $G / G_{0}$ as a function of the injection energy for a metallic graphene ribbon with 1625 dimer lines, with a series of 1,10 , and 100 tunnel barriers (orthogonal to the ribbon edges) with different Lorentzian profile and with different interbarrier distances. (b) Corresponding behavior of the Fano factor as a function of the injection energy.

the semiconducting ribbon, here for $E=0$ the conductance is not zero, but equal to the conductance quantum $G_{0}$, since the lowest-order mode is already able to propagate in the ribbon and transmits perfectly through the orthogonal barriers. As a consequence, the Fano factor vanishes: since there is unit transmission probability, each crossing event is hindered only by the presence of other electrons crossing the device at that moment; therefore, crossing events are perfectly correlated, the flow is regular, and no shot noise is present. Similarly, when the number of barriers is very high, only the lowestorder mode, which passes unaltered through the device, is able to propagate; therefore, the conductance becomes equal to $G_{0}$ and, since the mode is perfectly transmitted, the Fano factor vanishes.

In Fig. 7(a) and Fig. 7(b), instead, we report the conductance and Fano factor behavior, respectively, as a function of energy for the metallic armchair ribbon with 1625 dimer lines in the presence of a series of unevenly spaced (with random interbarrier distances between $150 \mathrm{~nm}$ and $250 \mathrm{~nm}$ ) different Lorentzian barriers with a $0.3 \mathrm{eV}$ peak, but with half-width at half-maximum randomly extracted between $2.5 \mathrm{~nm}$ and $37.5 \mathrm{~nm}$. In the panel (c) of Fig. 6 we show a color map of the transmission probability of the individual modes as a function of the injection energy and of the mode index. Similarly to what we have seen in the case of the semiconducting ribbon, the main difference with respect to the case of identical barriers is the disappearance, when several barriers are present, of resonant tunneling, and thus of the peaks in the transmission 

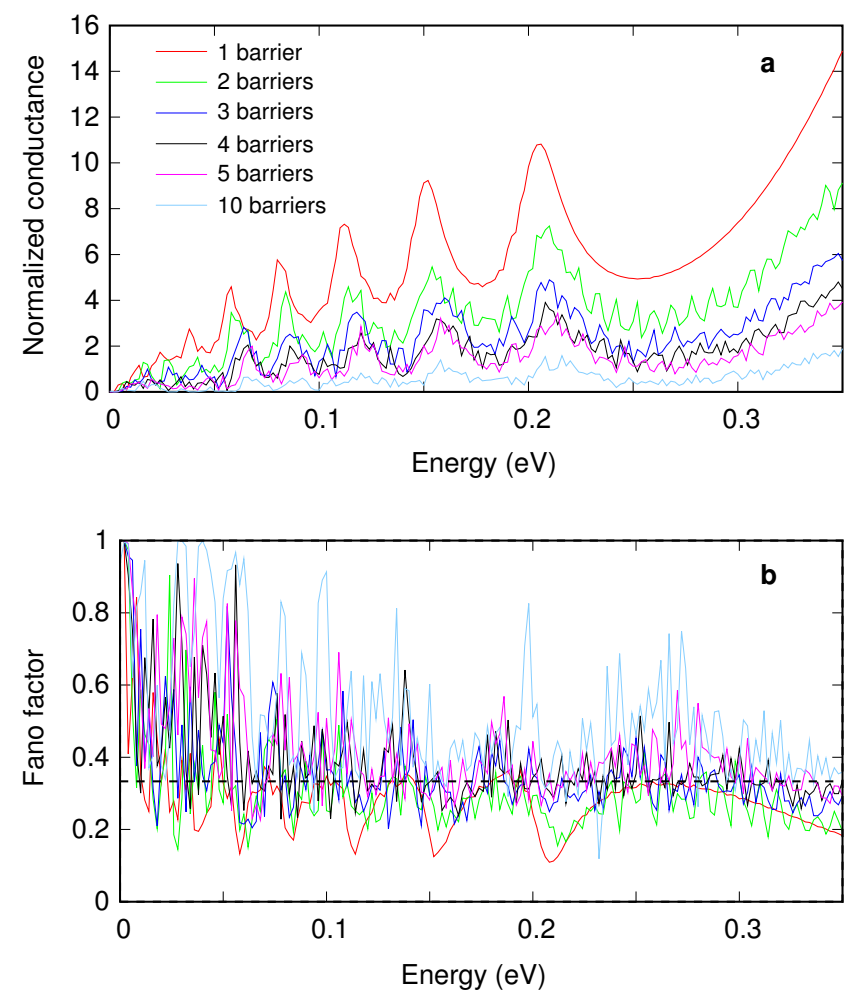

Fig. 8. (a) Normalized conductance $G / G_{0}$ as a function of the injection energy for a semiconducting graphene ribbon with 1626 dimer lines, with a series of $1,2,3,4,5$, and 10 tunnel barriers (tilted by $60^{\circ}$ with respect to the ribbon edges) with identical Lorentzian profile and with different interbarrier distances. (b) Corresponding behavior of the Fano factor as a function of the injection energy (the dashed thick horizontal line represents the $1 / 3$ value).

behavior as a function of energy. Since the barriers are different, their quasi-localized states have different energies $\mathcal{E}_{i, m}$, and thus it is not possible to have resonant tunneling through all the barriers at the same energy. Therefore, the behavior as a function of energy is more uniform, as we notice in the figures.

Finally, we have analyzed the case in which the barriers are still parallel, but are tilted by a certain angle with respect to the ribbon edges (instead of being orthogonal to them). In particular, we have considered Lorentzian barriers with a $0.3 \mathrm{eV}$ peak, a half-width at half-maximum equal to $20 \mathrm{~nm}$, a $60^{\circ}$ tilt angle with respect to the ribbon edges, and a distance along $x$ (the longitudinal direction of the ribbon) between the barrier maxima randomly distributed between $150 \mathrm{~nm}$ and $250 \mathrm{~nm}$. We report the behavior of the conductance and of the Fano factor as a function of energy, in the case of identical barriers, for a semiconducting ribbon with 1626 dimer lines in Fig. 8, and for a metallic ribbon with 1625 dimer lines in Fig. 9. The behavior is qualitatively similar to that observed for the case of barriers orthogonal to the ribbon edges, with Klein tunneling and resonant tunneling still playing their role. However, in this case a particularly interesting feature is observed. For a significant range of energies and of barrier numbers, the Fano factor approaches the value $1 / 3$ characteristic of diffusive transport. We explain these results in the following way. Diffusive transport regime can be reached when the device length is much greater than
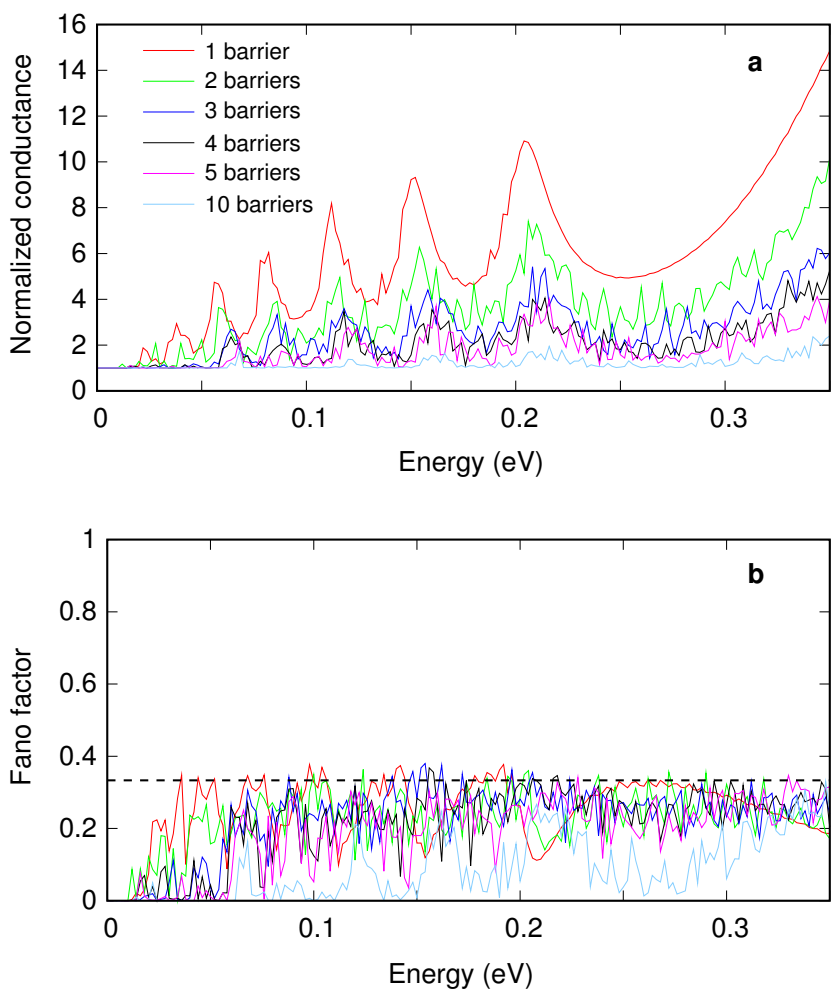

Fig. 9. (a) Normalized conductance $G / G_{0}$ as a function of the injection energy for a metallic graphene ribbon with 1625 dimer lines, with a series of $1,2,3,4,5$, and 10 tunnel barriers (tilted by $60^{\circ}$ with respect to the ribbon edges) with identical Lorentzian profile and with different interbarrier distances. (b) Corresponding behavior of the Fano factor as a function of the injection energy (the dashed thick horizontal line represents the $1 / 3$ value).

the mean free path and much less than the localization length. In the case of barriers orthogonal to the ribbon edges, each mode impinging against the barriers is partially transmitted and partially reflected, but is not converted into another mode: the transmission matrix of the structure is diagonal and the device can be seen as a collection of one-mode systems. Therefore, the localization length, which is of the order of the number of propagating modes times the mean free path, is similar to the mean free path and the diffusive regime can not be reached. On the contrary, a set of tilted barriers mixes the modes propagating through the device: in this case the device is a true many-mode system, the localization length is much greater than the mean free path, and diffusive transport can take place.

We have also noticed that the observed effects survive even in the presence of a low level of disorder (as we have shown in Ref. [12] for the case of barriers with a rectangular profile).

\section{CONCLUSIONS}

We have analyzed the behavior of an armchair graphene ribbon with a series of unevenly spaced, parallel potential barriers with a smooth, Lorentzian profile. We have found that this structure (which could be practically achieved placing a series of top gates at a certain distance from the graphene ribbon) can behave as mode and (in the case of identical barriers) energy filter. This device could be usefully exploited in all those cases, such as interference-based or low-noise 
devices, in which a pre-selection of the modes or of the energy components of the input signal is necessary or convenient for a good operation of a graphene-based system. In the future, our simulator could be improved performing a self-consistent transport calculation of the overall structure and including all the details of a practical implementation, and could be adopted to identify the optimal material and geometric parameters for the design and fabrication of the actual device.

\section{ACKNOWLEDGMENT}

This work was partially supported by the Italian Ministry of Education and Research (MIUR) in the framework of the CrossLab project (Departments of Excellence).

\section{REFERENCES}

[1] K. S. Novoselov, A. K. Geim, S. V. Morozov, D. Jiang, Y. Zhang, S. V. Dubonos, I. V. Grigorieva, and A. A. Firsov, "Electric Field Effect in Atomically Thin Carbon Films," Science, vol. 306, pp. 666-669, 2004, DOI: 10.1126/science.1102896.

[2] K. S. Novoselov, A. K. Geim, S. V. Morozov, D. Jiang, M. I. Katsnelson, I. V. Grigorieva, S. V. Dubonos, and A. A. Firsov, "Twodimensional gas of massless Dirac fermions in graphene," Nature, vol. 438, pp. 197-200, 2005, DOI: 10.1038/nature04233.

[3] A. H. Castro Neto, F. Guinea, N. M. R. Peres, K. S. Novoselov and A. K. Geim, "The electronic properties of graphene," Rev. Mod. Phys., vol. 81, pp. 109-162, 2009, DOI: 10.1103/RevModPhys.81.109.

[4] M. I. Katsnelson, Graphene: Carbon in Two Dimensions. Cambridge, UK: Cambridge University Press, 2012, ISBN: 0521195403.

[5] L. E. F. Foa Torres, S. Roche, and J.-C. Charlier, Introduction to Graphene-Based Nanomaterials: From Electronic Structure to Quantum Transport. Cambridge, UK: Cambridge University Press, 2020, ISBN: 1107030838.

[6] T. Enoki and T. Ando, Physics and Chemistry of Graphene: Graphene to Nanographene. Singapore, Singapore: Jenny Stanford Publishing, 2020, ISBN: 9814800384

[7] P. Avouris and C. Dimitrakopoulos, "Graphene: Synthesis and applications," Mater. Today, vol. 15, pp. 86-97, 2012, DOI: 10.1016/S13697021(12)70044-5.

[8] A. C. Ferrari, F. Bonaccorso, V. Fa'lko, K. S. Novoselov, S. Roche, P. Bøggild, S. Borini, F. H. L. Koppens, V. Palermo, N. Pugno, J. A. Garrido, R. Sordan, A. Bianco, L. Ballerini, M. Prato, E. Lidorikis, J. Kivioja, C. Marinelli, T. Ryhänen, A. Morpurgo, J. N. Coleman, V. Nicolosi, L. Colombo, A. Fert, M. Garcia-Hernandez, A. Bachtold, G. F. Schneider, F. Guinea, C. Dekker, M. Barbone, Z. Sun, C. Galiotis, A. N. Grigorenko, G. Konstantatos, A. Kis, M. Katsnelson, L. Vandersypen, A. Loiseau, V. Morandi, D. Neumaier, E. Treossi, V. Pellegrini, M. Polini, A. Tredicucci, G. M. Williams, B. H. Hong, J.-H. Ahn, J. M. Kim, H. Zirath, B. J. van Wees, H. van der Zant, L. Occhipinti, A. Di Matteo, I. A. Kinloch, T. Seyller, E. Quesnel, X. Feng, K. Teo, N. Rupesinghe, P. Hakonen, S. R. T. Neil, Q. Tannock, T. Löfwander, and J. Kinaret, "Science and technology roadmap for graphene, related two-dimensional crystals, and hybrid systems," Nanoscale, vol. 7, pp. 4598-4810, 2015, DOI: 10.1039/C4NR01600A.

[9] K. S. Novoselov, E. McCann, S. V. Morozov, V. I. Fal'ko, M. I. Katsnelson, U. Zeitler, D. Jiang, F. Schedin, and A. K. Geim, "Unconventional quantum Hall effect and Berry's phase of $2 \pi$ in bilayer graphene," Nat. Phys., vol. 2, pp. 177-180, 2006, DOI: 10.1038/nphys245.

[10] V. V. Cheianov, V. Fal'ko, and B. L. Altshuler, "The focusing of electron flow and a Veselago lens in graphene p-n junctions," Science, vol. 315, pp. 1252-1255 (2007), DOI: 10.1126/science.1138020.

[11] M. R. Connolly, R. K. Puddy, D. Logoteta, P. Marconcini, M. Roy, J. P. Griffiths, G. A. C. Jones, P. A. Maksym, M. Macucci, and C. G. Smith, "Unraveling Quantum Hall Breakdown in Bilayer Graphene with Scanning Gate Microscopy," Nano Lett., vol. 12, pp. 5448-5454, 2012, DOI: $10.1021 / \mathrm{n} 13015395$.

[12] P. Marconcini and M. Macucci, "Geometry-dependent conductance and noise behavior of a graphene ribbon with a series of randomly spaced potential barriers," J. Appl. Phys., vol. 125, p. 244302, 2019, DOI: $10.1063 / 1.5092512$.

[13] P. Marconcini and M. Macucci, "Symmetry-dependent transport behavior of graphene double dots," J. Appl. Phys., vol. 114, p. 163708, 2013, DOI: $10.1063 / 1.4827382$.
[14] P. Marconcini and M. Macucci, "Envelope-function based transport simulation of a graphene ribbon with an antidot lattice," IEEE Trans. Nanotechnol., vol. 16, pp. 534-544, 2017, DOI: 10.1109/TNANO.2016.2645663.

[15] J. Tworzydło, B. Trauzettel, M. Titov, A. Rycerz, and C. W. J. Beenakker, "Sub-Poissonian shot noise in graphene," Phys. Rev. Lett., vol. 96, p. 246802, 2006, DOI: 10.1103/PhysRevLett.96.246802.

[16] B. Pellegrini, P. Marconcini, M. Macucci, G. Fiori, and G. Basso, "Carrier density dependence of $1 / \mathrm{f}$ noise in graphene explained as a result of the interplay between band-structure and inhomogeneities," J. Stat. Mech.: Theory Exp., vol. 2016, p. 054017, 2016, DOI: 10.1088/17425468/2016/05/054017.

[17] M. Macucci and P. Marconcini, "Theoretical comparison between the flicker noise behavior of graphene and of ordinary semiconductors," $J$. Sensors, vol. 2020, p. 2850268, 2020, DOI: 10.1155/2020/2850268.

[18] G. R. Bhimanapati, Z. Lin, V. Meunie, Y. Jung, J. Cha, S. Das, D. Xiao, Y. Son, M. S. Strano, V. R. Cooper, L. Liang, S. G. Louie, E. Ringe, W. Zhou, S. S. Kim, R. R. Naik, B. G. Sumpter, H. Terrones, F. Xia, Y. Wang, J. Zhu, D. Akinwande, N. Alem, J. A. Schuller, R. E. Schaak, M. Terrones, and J. A. Robinson, "Recent Advances in Two-Dimensional Materials beyond Graphene," ACS Nano, vol. 9, pp. 11509-11539 (2015), DOI: 10.1021/acsnano.5b05556.

[19] P. Miró, M. Audiffreda, and T. Heine, "An atlas of two-dimensional materials," Chem. Soc. Rev., vol. 43, pp. 6537-6554, 2014, DOI: $10.1039 / \mathrm{C} 4 \mathrm{CS} 00102 \mathrm{H}$.

[20] L. Jiang, P. Marconcini, M. S. Hossian, W. Qiu, R. J. Evans, M. Macucci, and S. Skafidas, "A tight binding and $\mathrm{k} \cdot \mathrm{p}$ study of monolayer stanene," Sci Rep., vol. 7, p. 12069, 2017, DOI: 10.1038/s41598017-12281-y.

[21] A. K. Geim and K. S. Novoselov, "The rise of graphene," Nat. Mater, vol. 6, pp. 183-191, 2007, DOI: 10.1038/nmat1849.

[22] K. S. Novoselov, V. I. Fal'ko, L. Colombo, P. R. Gellert, M. G. Schwab, K. Kim, "A roadmap for graphene," Nature, vol. 490, pp. 192-200, 2012, DOI: 10.1038/nature11458.

[23] Y. Zhu, H. Ji, H.-M. Cheng, and R. S. Ruoff, "Mass production and industrial applications of graphene materials," Natl. Sci. Rev., vol. 5, pp. 90-101, 2018, DOI: 10.1093/nsr/nwx055.

[24] H. Raza, Graphene Nanoelectronics: Metrology, Synthesis, Properties and Applications. Berlin, Germany: Springer-Verlag, 2012, ISBN: 9783642204678 .

[25] R. Murali, Graphene Nanoelectronics: From Materials to Circuits. New York, NY: Springer, 2012, ISBN: 1461405475.

[26] Y. Wu, D. B. Farmer, F. Xia, and P. Avouris, "Graphene Electronics: Materials, Devices, and Circuits," Proc. IEEE, vol. 101, pp. 1620-1637, 2013, DOI: 10.1109/JPROC.2013.2260311.

[27] K. C. Yung, W. M. Wu, M. P. Pierpoint, and F. V. Kusmartsev "Introduction to graphene electronics - a new era of digital transistors and devices," Contemp. Phys., vol. 54, pp. 233-251, 2013, DOI: 10.1080/00107514.2013.833701

[28] T. Das, B. K. Sharma, A. K. Katiyar, and J.-H. Ahn, "Graphene-based flexible and wearable electronics," J. Semicond., vol. 39, p. 011007 , 2018, DOI: 10.1088/1674-4926/39/1/011007.

[29] P. Marconcini, A. Cresti, F. Triozon, G. Fiori, B. Biel, Y.-M. Niquet, M. Macucci, and S. Roche, "Atomistic Boron-Doped Graphene FieldEffect Transistors: A Route toward Unipolar Characteristics," ACS Nano, vol. 6, pp. 7942-7947, 2012, DOI: 10.1021/nn3024046.

[30] P. Marconcini, A. Cresti, and S. Roche, "Effect of the Channel Length on the Transport Characteristics of Transistors Based on BoronDoped Graphene Ribbons," Materials, vol. 11, p. 667, 2018, DOI: 10.3390/ma11050667.

[31] F. Schwierz, "Graphene Transistors," Nature Nanotech, vol. 5, pp. 487496, 2010, DOI: 10.1038/nnano.2010.89.

[32] F. Schwierz, "Graphene Transistors: Status, Prospects, and Problems," Proc. IEEE, vol. 101, pp. 1567-1584, 2013, DOI: 10.1109/JPROC.2013.2257633.

[33] E. W. Hill, A. Vijayaragahvan, and K. Novoselov, "Graphene Sensors," IEEE Sens. J., vol. 11, pp. 3161-3170, 2011, DOI: 10.1109/JSEN.2011.2167608.

[34] P. Marconcini, M. Macucci, "The k.p method and its application to graphene, carbon nanotubes and graphene nanoribbons: the Dirac equation," Riv. Nuovo Cimento, vol. 34, pp. 489-584, 2011, DOI: 10.1393/ncr/i2011-10068-1.

[35] M. I. Katsnelson, K. S. Novoselov, and A. K. Geim, "Chiral tunnelling and the Klein paradox in graphene," Nat. Phys., vol. 2, pp. 620-625, 2006, DOI: $10.1038 /$ nphys 384 . 
[36] C. W. J. Beenakker, "Colloquium: Andreev reflection and Klein tunneling in graphene," Rev. Mod. Phys., vol. 80, pp. 1337-1354, 2008, DOI: $10.1103 /$ RevModPhys.80.1337.

[37] A. F. Young, P. Kim, "Quantum interference and Klein tunnelling in graphene heterojunctions," Nature Phys., vol. 5, pp. 222-226, 2009, DOI: $10.1038 /$ nphys 1198 .

[38] V. Nam Do, V. Hung Nguyen, P. Dollfus, and A. Bournel, "Electronic transport and spin-polarization effects of relativisticlike particles in mesoscopic graphene structures," J. Appl. Phys., vol. 104, p. 063708, 2008, DOI: $10.1063 / 1.2980045$.

[39] V. Hung Nguyen, A. Bournel, and P. Dollfus, "Resonant tunneling structures based on epitaxial graphene on SiC," Semicond. Sci. Technol., vol. 26, p. 125012, 2011, DOI: 10.1088/0268-1242/26/12/125012.

[40] P. E. Allain and J. N. Fuchs, "Klein tunneling in graphene: Optics with massless electrons," Eur. Phys. J. B, vol. 83, pp. 301-317, 2011, DOI: 10.1140/epjb/e2011-20351-3.

[41] I. Rodríguez-Vargas, J. Madrigal-Melchor, and O. Oubram, "Resonant tunneling through double barrier graphene systems: A comparative study of Klein and non-Klein tunneling structures," J. Appl. Phys., vol. 112, p. 073711, 2012, DOI: 10.1063/1.4757591.

[42] J. M. Pereira Jr., F. M. Peeters, A Chaves, and G. A. Farias, "Klein tunneling in single and multiple barriers in graphene," Semicond. Sci. Technol., vol. 25, p. 033002, 2010, DOI: 10.1088/0268-1242/25/3/033002.

[43] C.-H. Park, Y.-W. Son., L. Yang, M. L. Cohen, and S. G. Louie, "Electron Beam Supercollimation in Graphene Superlattices," Nano Lett., vol. 8, pp. 2920-2924, 2008, DOI: 10.1021/nl801752r

[44] D. Dragoman and M. Dragoman, "Negative differential resistance of electrons in graphene barrier," Appl. Phys. Lett., vol. 90, p. 143111, 2007, DOI: 10.1063/1.2719670.

[45] R. Zhu and Y. Guo, "Shot noise in the graphene-based doublebarrier structures," Appl. Phys. Lett., vol. 91, p. 252113, 2007, DOI: $10.1063 / 1.2825571$.

[46] T. Tudorovskiy, K. J. A. Reijnders, and M. I. Katsnelson, "Chiral tunneling in single-layer and bilayer graphene," Phys. Scr, vol. T146, p. 014010, 2012, DOI: 10.1088/0031-8949/2012/T146/014010.

[47] N. T. T. Nguyen, D. Q. To, and V. L. Nguyen, "A model for ballistic transport across locally gated graphene bipolar junctions," $J$. Phys. Condens. Matter, vol. 26, p. 015301, 2014, DOI: 10.1088/09538984/26/1/015301.

[48] H. C. Nguyen and V. L. Nguyen, "Tunneling of Dirac electrons through one-dimensional potentials in graphene: a $T$-matrix approach," $J$. Phys.: Condens. Matter, vol. 21, p. 045305, 2009, DOI: 10.1088/09538984/21/4/045305.

[49] L. Brey and H. A. Fertig, "Emerging zero modes for graphene in a periodic potential," Phys. Rev. Lett., vol. 103, p. 046809, 2009, DOI: 10.1103/PhysRevLett.103.046809.

[50] P. Burset, A. Levy Yeyati, L. Brey, and H. A. Fertig, "Transport in superlattices on single-layer graphene," Phys. Rev. B, vol. 83, p. 195434, 2011, DOI: 10.1103/PhysRevB.83.195434.

[51] M. Barbier, P. Vasilopoulos, and F. M. Peeters, "Extra Dirac points in the energy spectrum for superlattices on single-layer graphene," Phys. Rev. B, vol. 81, p. 075438, 2010, DOI: 10.1103/PhysRevB.81.075438.

[52] M. Barbier, P. Vasilopoulos, and F. M. Peeters, "Single-layer and bilayer graphene superlattices: collimation, additional Dirac points and Dirac lines," Phil. Trans. R. Soc. A, vol. 368, pp. 5499-5524, 2010, DOI: $10.1098 /$ rsta.2010.0218.

[53] S. Dubey, V. Singh, A. K. Bhat, P. Parikh, S. Grover, R. Sensarma, V. Tripathi, K. Sengupta, and M. M. Deshmukh, "Tunable superlattice in graphene to control the number of Dirac points," Nano Lett., vol. 13, pp. 3990-3995, 2013, DOI: 10.1021/n14006029.

[54] G. J. Xu, X. G. Xu, B. H. Wu, J. C. Cao, and C. Zhang, "The resonant tunneling through a graphene multiquantum well system," J. Appl. Phys., vol. 107, p. 123718, 2010, DOI: 10.1063/1.3445782.

[55] Y. O. Klymenko and O. Shevtsov, "Quantum transport in armchair graphene ribbons: analytical tight-binding solutions for propagation through step-like and barrier-like potentials," Eur. Phys. J. B, vol. 69, pp. 383-388, 2009, DOI: 10.1140/epjb/e2009-00178-3.

[56] Y. Klymenko, L. Malysheva, and A. Onipko "Electron transmission through step- and barrier-like potentials in graphene ribbons," Phys. Stat. Sol. (b), vol. 245, pp. 2181-2184, 2008, DOI: $10.1002 / p s s b .200879637$.

[57] A. Cresti, G. Grosso, and G. Pastori Parravicini, "Numerical study of electronic transport in gated graphene ribbons," Phys. Rev. B, vol. 76 , p. 205433, 2007, DOI: 10.1103/PhysRevB.76.205433.

[58] O. Roslyak, A. Iurov, G. Gumbs, and D. Huang, "Unimpeded tunneling in graphene nanoribbons," J. Phys.: Cond. Matt., vol. 22, p. 165301, 2010, DOI: 10.1088/0953-8984/22/16/165301.
[59] J. H. Oh and D. Ahn, "Klein Tunnelling Through an Oblique Barrier in Graphene Ribbons," J. Korean Phy. Soc., vol. 59, pp. 2786-2791, 2011. DOI: $10.3938 / \mathrm{jkps} .59 .2786$

[60] E. D. Herbschleb, R. K. Puddy, P. Marconcini, J. P. Griffiths, G. A. C. Jones, M. Macucci, C. G. Smith, and M. R. Connolly, "Direct imaging of coherent quantum transport in graphene p-n-p junctions," Phys. Rev. B, vol. 92, p. 125414, 2015, DOI: 10.1103/PhysRevB.92.125414.

[61] P. Marconcini and M. Macucci, "Numerical analysis of the resistance behavior of an electrostatically-induced graphene double junction," $J$. Comput. Electron., vol. 14, p. 653-660, 2015, DOI: 10.1007/s10825015-0701-5.

[62] P. Marconcini and M. Macucci, "Approximate calculation of the potential profile in a graphene-based device," IET Circ. Device Syst., vol. 9, pp. 30-38, 2015, DOI: 10.1049/iet-cds.2014.0003.

[63] D. Logoteta, P. Marconcini, C. Bonati, M. Fagotti, and M. Macucci, "High-performance solution of the transport problem in a graphene armchair structure with a generic potential," Phys. Rev. E, vol. 89, p. 063309, 2014, DOI: 10.1103/PhysRevE.89.063309.

[64] H. B. Nielsen and M. Ninomiya, "A no-go theorem for regularizing chiral fermions," Phys. Lett. B, vol. 105, pp. 219-223, 1981, DOI: 10.1016/0370-2693(81)91026-1.

[65] P. Marconcini, "The role of the choice of the physical model in the optimization of nanoelectronic device simulators," International Journal of Circuits, Systems and Signal Processing, vol. 7, pp. 173180, 2013.

[66] M. Fagotti, C. Bonati, D. Logoteta, P. Marconcini, and M. Macucci, "Armchair graphene nanoribbons: $\mathcal{P} \mathcal{T}$-symmetry breaking and exceptional points without dissipation," Phys. Rev. B, vol. 83, p. 241406(R), 2011, DOI: 10.1103/PhysRevB.83.241406.

[67] P. Marconcini, D. Logoteta, and M. Macucci, "Sinc-based method for an efficient solution in the direct space of quantum wave equations with periodic boundary conditions," J. Appl. Phys., vol. 114, p. 173707, 2013, DOI: $10.1063 / 1.4828875$.

[68] S. Datta, Electronic Transport in Mesoscopic Systems, Cambridge, UK: Cambridge University Press, 1997, ISBN: 9780521599436.

[69] R. Landauer, "Spatial variation of currents and fields due to localized scatterers in metallic conduction," IBM J. Res. Dev., vol. 1, pp. 223231, 1957, DOI: $10.1147 /$ rd.13.0223

[70] M. Büttiker, Y. Imry, R. Landauer, and S. Pinhas, "Generalized many-channel conductance formula with application to small rings," Phys. Rev. B, vol. 31, pp. 6207-6215, 1985, DOI: 10.1103/PhysRevB.31.6207.

[71] M. Büttiker, "Scattering theory of thermal and excess noise in open conductors," Phys. Rev. Lett., vol. 65, pp. 2901-2904, 1990, DOI 10.1103/PhysRevLett.65.2901.

[72] E. Anderson, Z. Bai, C. Bischof, S. Blackford, J. Demmel, J. Dongarra, J. Du Croz, A. Greenbaum, S. Hammarling, A. McKenney, and D. Sorensen, LAPACK Users' Guide. Philadelphia, PA: Society for Industrial and Applied Mathematics, 1999, ISBN: 0898714478.

[73] V.-Hung Nguyen, "Electronic transport and spin-polarization effects in graphene nanostructures," Ph.D. Thesis, Université de Paris-Sud, Ecole doctorale Sciences et Technologies de l'Information, des Télćommunications et des Systèmes, Orsay, Essonne, France, 2010.

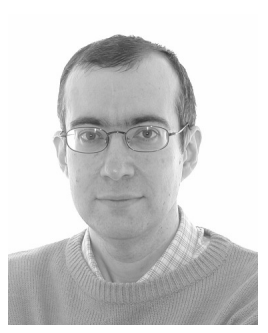

Paolo Marconcini Paolo Marconcini received the Master's (summa cum laude) degree in Electrical Engineering and the Ph.D. degree in Electrical and Computer Engineering from the Università di Pisa, Pisa, Italy, in 2002 and 2006, respectively. From 2006, he has been a Post-Doctoral Researcher and then Tenure-Track Researcher with the Università di Pisa, where he is now Associate Professor. He has authored over 100 papers in international journals and proceedings and has been involved in over ten national and international research projects. His current research interests include the study of nanoelectronic devices based on semiconductor heterostructures, nanowires, carbon nanotubes, graphene and two-dimensional materials, and of electronic systems for transportation.

Creative Commons Attribution License 4.0 (Attribution 4.0 International, CC BY 4.0)

This article is published under the terms of the Creative Commons Attribution License 4.0 https://creativecommons.org/licenses/by/4.0/deed.en_US 